\title{
Investigation of Low Carbohydrate Diet for Type 2 Diabetes Mellitus with Elevated Immunoreactive Insulin
}

\author{
Hiroshi Bando $^{1,2^{*}}$, Koji Ebe ${ }^{2,3}$, Tetsuo Muneta ${ }^{2,4}$, Masahiro Bando ${ }^{5}$ and Yoshikazu Yonei ${ }^{6}$ \\ ${ }^{1}$ Tokushima University / Medical Research, Tokushima, Japan \\ ${ }^{2}$ Japan Low Carbohydrate Diet Promotion Association, Kyoto, Japan \\ ${ }^{3}$ Takao Hospital, Kyoto, Japan \\ ${ }^{4}$ Muneta Maternity Clinic, Chiba, Japan \\ ${ }^{5}$ Department of Nutrition and Metabolism, Institute of Biomedical Sciences, Tokushima University Graduate School, \\ Tokushima, Japan \\ ${ }^{6}$ Anti-Aging Medical Research Center, Graduate School of Life and Medical Sciences, Doshisha University, Kyoto, Japan
}

"Corresponding author: Bando H, MD, PhD, FACP, Tokushima University /Medical Research, Nakashowa 1-61, Tokushima 770-0943 Japan, Tel: +81-90-3187-2485; E-mail: pianomed@bronze.ocn.ne.jp

Received: August 19, 2018; Accepted: August 28, 2018; Published: August 31, 2018

\begin{abstract}
Background: There are discussion of Calorie Restriction (CR) and Low Carbohydrate Diet (LCD). Authors and colleagues have continued research concerning LCD, CR and Morbus (M) value. Furthermore, this study also included homeostasis model assessment (HOMA).

Subjects and Methods: Subjects enrolled were 40 type 2 diabetes mellitus (T2DM) cases with more than $10 \mu \mathrm{U} / \mathrm{mL}$ of fasting immunoreacitve insulin (IRI). Methods included fundamental tests such as glucose, IRI, HOMA-R, HOMA- $\beta$, average blood glucose on day 2 and 14 during CR and LCD meal.

Results: Current data were in the following: average age $56.8 \pm 12.3$ years old, median values are HbA1c 7.2\%, fasting glucose $141 \mathrm{mg} / \mathrm{dL}$, IRI 13.1 $\mu \mathrm{U} / \mathrm{mL}$, HOMA-R 4.6, HOMA-ß 57.1. Median values on day 2 vs 14 were average blood glucose 160 vs 120 mg/dL, M value 37.1 vs 7.4, Triglyceride $137 \mathrm{vs} 97 \mathrm{mg} / \mathrm{dL}$, respectively. M value showed significant correlation with HOMA-R $(\mathbf{p}<0.05)$ and HOMA- $\beta(\mathbf{p}<0.01)$.

Discussion and Conclusion: The results suggested that LCD showed clinical effect for decreasing of glucose and $M$ value in glucose variability and those patients would have insulin resistance and decreased $\beta$ cell function. Furthermore, these findings would become basal and useful data for future research in this field.
\end{abstract}

Keywords: Morbus value (M value); Type 2 diabetes mellitus (T2DM); Low carbohydrate diet (LCD); Daily profile of blood glucose; Homeostasis model assessment of insulin resistance (HOMA-R); Homeostasis model assessment of $\beta$ cell function (HOMA- $\beta)$

Citation: Bando H, Ebe K, Muneta T, et al. Investigation of Low Carbohydrate Diet for Type 2 Diabetes Mellitus with Elevated Immunoreactive Insulin. Clin Case Rep Open Access. 2018;1(1):103.

(C)2018 Yumed Text. 
www.yumedtext.com | August-2018

\section{Introduction}

In clinical practice, diabetes has been one of the crucial problems to be solved in the world. The management for diabetes has been on discussion concerning diagnosis and treatment. In recent years, there has been some controversy on successive discussion about the recommended HbA1c in International Diabetes Federation (IDF), American Diabetes Association (ADA) and American College of Physicians (ACP) [1-3]. Thus, applicable control of diabetic variability has been important to be argued [4].

Furthermore, the nutrition therapy for diabetes has been the fundamental treatment for diabetes and Metabolic syndrome (Met-S). Historically, the method of Calorie Restriction (CR) has been the main principle for diet therapy widely, but in 1990' Bernstein et al. started to apply Low Carbohydrate Diet (LCD) [5]. The efficacy of LCD for reduction of weight and decrease of blood glucose has been rather recognized and known. In concession, Shai et al. [6] showed the data of the comparison of the efficacy of LCD, Mediterranean, Low-Fat Diet [6,7]. Such discussion has been continued for long years between CR and LCD [8,9].

Meanwhile, in Japan, authors and co-researchers have introduced and developed LCD so far. The methods have included educational seminars, medical journals and books and presenting in medical society [10,11]. We have also investigated medical papers concerning the physiological increased value of Ketone Bodies $(\mathrm{KB})$ in the circumstances of fetus, placenta, newborn and mother [12]. Further, we have developed the prevalence of LCD by the activity of Japan LCD promotion association [13]. The recommended simple way of LCD would be summarized in 3 types. They are super LCD, moderate LCD and petite LCD meals [14].

In this study, we compared the glucose variability on CR diet and LCD, and calculated the difference of average blood glucose and Morbus (M) value. Furthermore, we investigated the correlation of biomarkers related to diabetes such as homeostasis model assessment of insulin resistance (HOMA-R) and homeostasis model assessment of $\beta$ cell function (HOMA- $\beta$ ).

\section{Subjects and Methods}

In this study, the enrolled subjects were 40 patients with Type 2 Diabetes Mellitus (T2DM). Subjects were diagnosed as diabetes, and admitted to the hospital for further evaluation and treatment of diabetes. As to the methods, we have already a formula protocol of diabetic evaluation. We decided to follow a certain criteria concerning this study.

i. From lots of diabetic patients studied, the subjects were diagnosed as T2DM and other specific type of diabetes were excluded. Further, subjects with fasting immunoreactive insulin (IRI) more than $10 \mu \mathrm{U} / \mathrm{mL}$ were included in this study.

ii. The patients were admitted for 2 weeks in the hospital. On the morning of day 2, blood samples for basal data were drawn after overnight fasting. Basal tests contain biomarkers such as complete blood count, liver, renal lipids and so on. Regarding diabetes, HbA1c, glucose, IRI, C-peptide, HOMA-R, HOMA- $\beta$, M value were measured and calculated for the study. 
www.yumedtext.com | August-2018

iii. According to our protocol test, the nutritional therapy was performed as follows. Subjects have daily meal. On day 1 and 2, CR meal contains protein $15 \%$, fat $25 \%$ and carbohydrate $60 \%$ with $1400 \mathrm{kcal} /$ day. This ratio is from the standard of the macronutrients due to Japan Diabetes Association [15].

iv. Subjects were provided LCD meal from day 3 to 14 with super LCD. The content has $12 \%$ of carbohydrate with $1400 \mathrm{kcal} / \mathrm{day}$. We have other two formulas, which are standard LCD with carbohydrate $26 \%$ and petite LCD with carbohydrate $40 \%$. These 3 types of LCD have been educated to people so far. In this study, all subjects were given only super LCD with $12 \%$ of carbohydrate.

v. Biomarkers related to diabetes were measured in day 2 and day 14. Blood samples in both days were drawn in the morning after overnight fasting. Both data and several correlations were compared and investigated. The reason of examination of day 2 is that day 1 is not adequate to check glucose variability due to unstable condition on the day of admission.

\subsection{Daily profile of blood glucose}

As regard to glucose metabolism, daily profile of blood glucose was studied on day 2 and on day 14. In each day, blood glucose levels were measured 7 times a day. Its clock times were 0800, 1000, 1200, 1400, 1700, 1900, 2200 h. After obtaining the data of blood glucose, average blood glucose a day and also the level of Morbus (M) value were calculated according to the formula equation [16,17].

\subsection{Morbus value}

One of the useful biomarker for glucose variability is the $\mathrm{M}$ value. It includes two important clinical factors. One expresses the average blood glucose level in a day, and another is the mean amplitude of glycemic excursions (MAGE) [16-18]. Thus, $\mathrm{M}$ value is simply one numerical value, which means the degree of elevated blood glucose and increased fluctuation of blood glucose. In mathematical aspect, $\mathrm{M}$ value is calculated as the method of logarithmic transformation. In other words, the significance of $\mathrm{M}$ value expresses the glucose deviation from the ideal glucose variability [17-19].

$M$ value includes the calculation of three steps. Firstly, $M=M^{B S}+M^{W}$ : $M$ value expresses the total of $M^{B S}$ and $M^{W}$. Secondary, $\mathrm{M}^{\mathrm{W}}$ expresses (maximum blood glucose - minimum glucose)/20. Tertiary, $\mathrm{M}^{\mathrm{BS}}$ is the mean of MBSBS. When these are summarized, MBSBS has been the individual M-value for each blood glucose, calculated as (absolute value of [10 $\times \log (\text { blood glucose level/120)] })^{3}$ [17-19]. Regarding the level of M value, clinical judgement would be used for general. M value would be that normal range is $<180$, borderline is from 180 to 320 , and abnormal range is $320<$.

\subsection{Statistical analysis}

As to current investigation, obtained data were revealed by mean and standard deviation. Moreover, several data were described as the median and quartile of $25 \% / 75 \%$. For comparison among some groups, boxplot method was used. It means the median and the quartile of $25 \% / 75$, maximum and minimum. Regarding the correlation with biomarkers, Spearman test was used for the correlation coefficients. We could utilize the computerized standard statistical tool for analytical evaluation [20]. 
www.yumedtext.com | August-2018

\section{Ethical Considerations}

This study was conducted in compliance with the ethical principles based upon the Declaration of Helsinki. Moreover, additional commentary was performed in the Ethical Guidelines for Medical Research in Humans and in accordance with the Good Clinical Practice (GCP), associated with the ongoing consideration to the protection of subjects' human rights. In addition, there was the "Ethical Guidelines for Epidemiology Research" by the Ministry of Education, Culture, Sports, Science and Technology and the Ministry of Health, Labor and Welfare.

Author and co-researchers had an ethical committee. For the discussion, physician, nurse, pharmacist and other experts in the legal specialty attended. As to current investigation, we discussed and confirmed that this investigation would be valid and agreed. Furthermore, the informed consents and written paper agreements have been obtained from the subjects. This study has been registered by National University Hospital Council of Japan (ID: \#R000031211).

\section{Results}

\subsection{Fundamental data}

Subjects ( $\mathrm{n}=40)$ enrolled showed the fundamental data (TABLE 1). Average age was $56.8 \pm 12.3$ years old with 57 years old in median. Median value of $\mathrm{HbA1c}$, fasting blood glucose and IRI was $7.2 \%, 141 \mathrm{mg} / \mathrm{dL}$ and $13.1 \mu \mathrm{U} / \mathrm{mL}$, respectively. Median value of HOMA-R and HOMA- $\beta$ was 4.6 and 57.1, respectively.

TABLE 1. Subject and basal data.

\begin{tabular}{|c|c|c|}
\hline & Mean \pm SD & median $[25 \%-75 \%]$ \\
\hline \multicolumn{3}{|l|}{ Subjects } \\
\hline number $(\mathrm{M} / \mathrm{F})$ & $40(17 / 23)$ & $40(17 / 23)$ \\
\hline age (years old) & $56.8 \pm 12.3$ & 57 [49-65] \\
\hline \multicolumn{3}{|l|}{ Glucose profile } \\
\hline HbA1c (\%) & $7.5 \pm 1.8$ & $7.2[6.1-8.1]$ \\
\hline fast glucose $(\mathrm{mg} / \mathrm{dL})$ & $147 \pm 35.5$ & 141 [121-158] \\
\hline fast IRI $(\mu \mathrm{U} / \mathrm{mL})$ & $14.4 \pm 4.2$ & $13.1[11.4-16.5]$ \\
\hline \multicolumn{3}{|l|}{ Homeostasis } \\
\hline HOMA-R & $5.0 \pm 1.7$ & $4.6[3.9-5.4]$ \\
\hline HOMA- $\beta$ & $74.8 \pm 44.8$ & $57.1[43.7-90.6]$ \\
\hline
\end{tabular}

\subsection{Changes in biomarkers}

In response to LCD for 2 weeks, several related biomarkers between day 2 and day 14 were compared (TABLE 2). Decreased median values from day 2 to day 14 were observed in average blood glucose, $\mathrm{M}$ value and Triglyceride, which were 160 vs $120 \mathrm{mg} / \mathrm{dL}, 37.1$ vs $7.4,137$ vs $97 \mathrm{mg} / \mathrm{dL}$, respectively. 
TABLE 2. Data of biomarkers between day 2 and day 14.

\begin{tabular}{|c|c|c|}
\hline & Mean \pm SD & median [25\%-75\%] \\
\hline Glucose metabolism & & \\
\hline Av. Glucose $(\mathrm{mg} / \mathrm{dL})$ & & $160[123-190]$ \\
\hline day 2 & $168 \pm 53.3$ & $120[106-145]$ \\
\hline day 14 & $128 \pm 30.7$ & $37.1[9.7-111]$ \\
\hline M value & $93.4 \pm 130$ & $120[3.9-13.3]$ \\
\hline day 2 & $16.4 \pm 28.2$ & $137[112-195]$ \\
\hline day 14 & & $97.0[76.5-124]$ \\
\hline Lipid metabolism & & \\
\hline Triglyceride(mg/dL) & $182 \pm 169$ & $51.0[43.0-57.5]$ \\
\hline day 2 & $110 \pm 48.3$ & $42.0[37.0-50.0]$ \\
\hline day 14 & & $121[97.8-141]$ \\
\hline HDL-C (mg/dL) & $51.7 \pm 11.2$ & $130[114-116]$ \\
\hline day 2 & $44.4 \pm 10.5$ & \\
\hline day 14 & $123 \pm 30.9$ & \\
\hline LDL-C (mg/dL) & $140 \pm 45.1$ & \\
\hline day 2 & & \\
\hline day 14 & & \\
\hline
\end{tabular}

\subsection{Comparison on day 2 and 14 in 4 groups}

Average blood glucose was decreased from day 2 to 14 in 4 groups (FIG. 1a). In the case M value, there were decreased value from day 2 to day 14 in group 2,3,4, while $\mathrm{M}$ value did not change much in group 1 (FIG. 1b). The level of triglyceride decreased from day 2 to 14 in 4 groups (FIG. 1c).

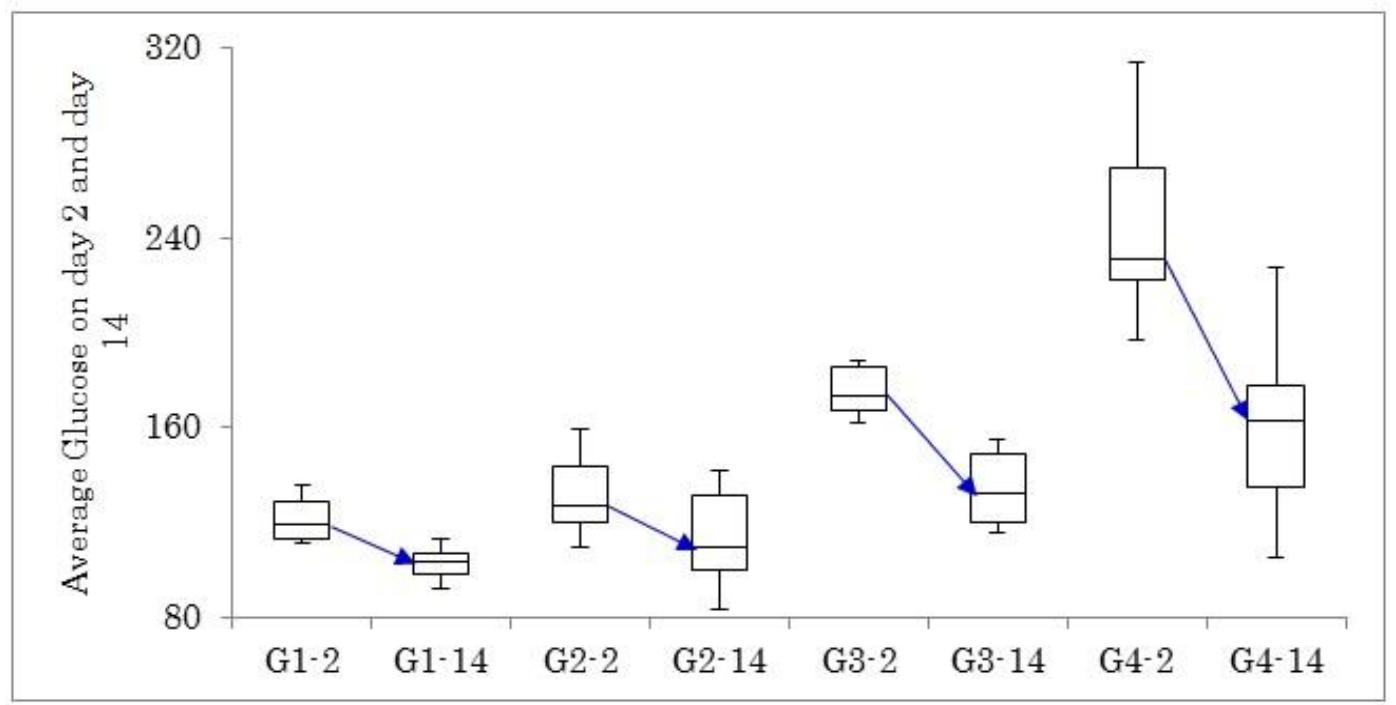

FIG. 1a. Average blood glucose decreased from day 2 to 14 in 4 groups. 
www.yumedtext.com | August-2018

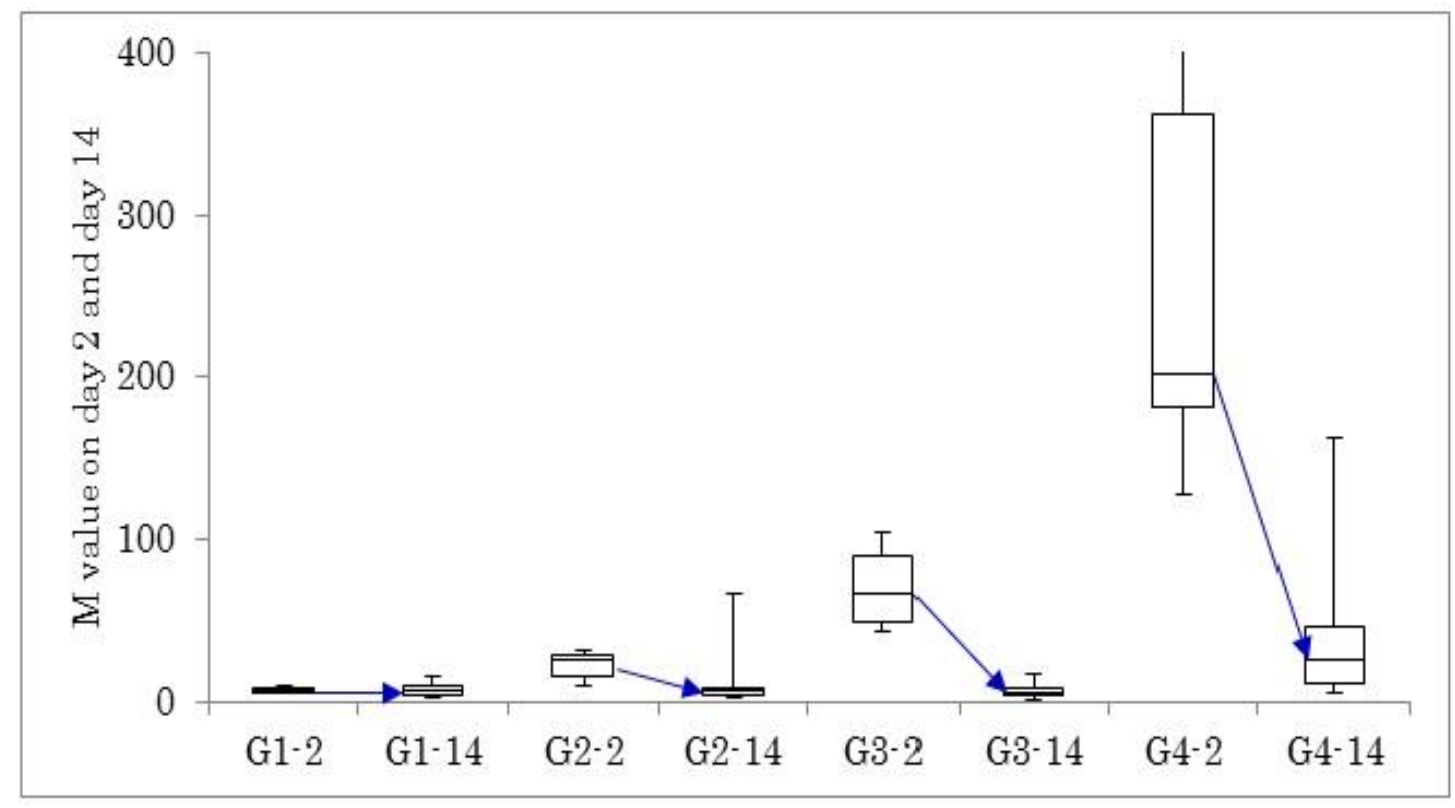

FIG. 1b. M value decreased from day 2 to 14 in 4 groups.

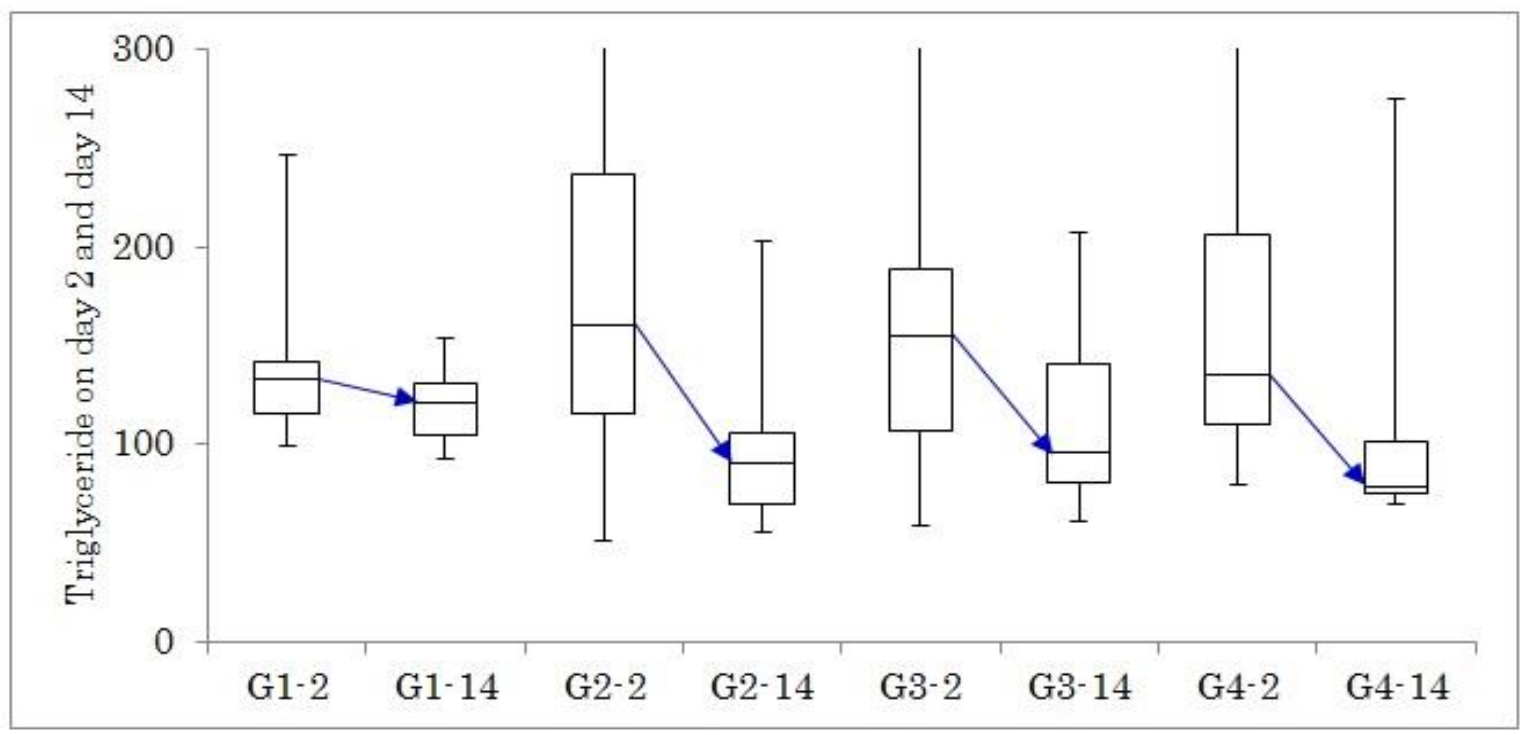

FIG. 1c. Triglyceride decreased from day 2 to 14 in 4 groups.

FIG. 1. Comparison of biomarkers on day 2 and 14 in 4 groups.

\subsection{Correlations of biomarkers between day 2 and 14}

Average glucose showed significant correlation between day 2 and $14(\mathrm{p}<0.01)$ (FIG. 2a). The regression curve was $y=$ $0.48 x+45.9$, indicating that average glucose decrease moderately in this period.

$\mathrm{M}$ value showed significant correlation between day 2 and 14 ( $\mathrm{p}<0.01)$ (FIG. 2b). The regression curve was $y=0.16 \mathrm{x}+1.2$, indicating that $\mathrm{M}$ value decrease remarkably in this period. 


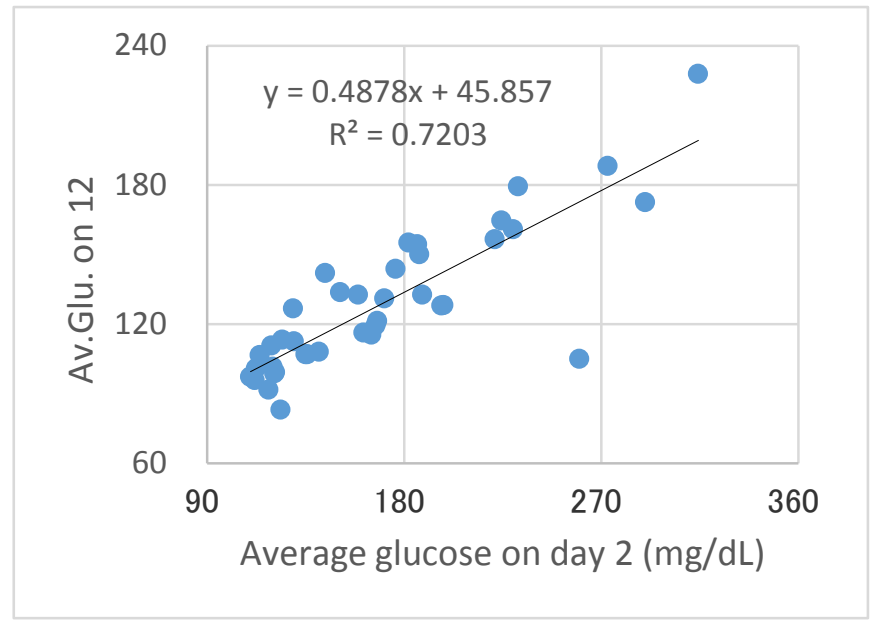

$2 a$

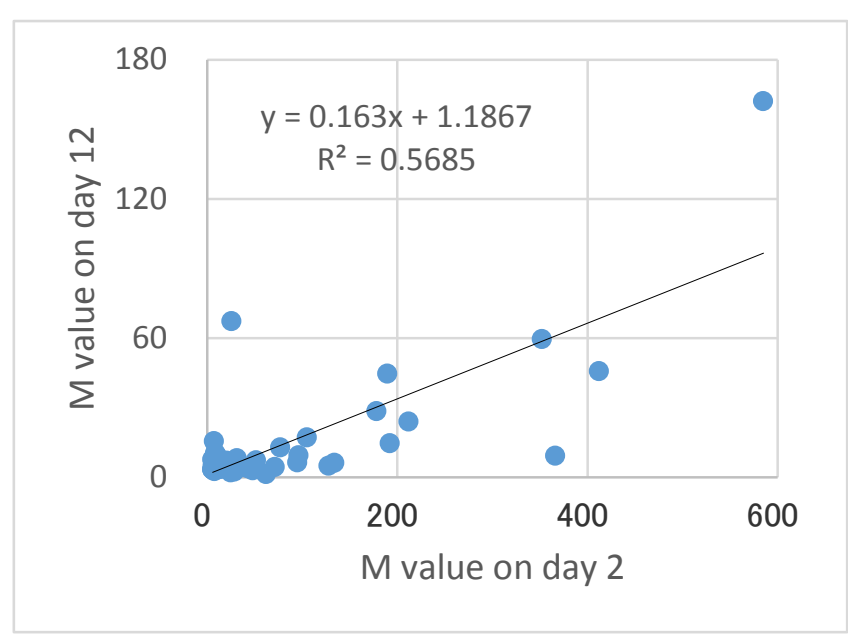

$2 b$

FIG. 2. Correlations of biomarkers between day 2 and 12. FIG. 2a. Average glucose showed significant correlation between day 2 and $14(\mathrm{p}<0.01)$. The regression curve was $0.48 \mathrm{x}+45.9$, indicating that average glucose decrease moderately in this period, FIG. 2b. M value showed significant correlation between day 2 and $14(\mathrm{p}<0.01)$. The regression curve was $0.16 \mathrm{x}+1.2$, indicating that $\mathrm{M}$ value decrease remarkably in this period.

\subsection{Categorization by $M$ value}

Subjects were divided into 4 groups due to the level of $M$ value. Each group includes 10 cases. The median level of $M$ value in group 1-4 was 7.0, 25.1, 66.7, and 202, respectively. From group 1 to group 4, the level of M value has increased remarkably. In 4 groups, average blood glucose and HbA1c were compared (FIG. 3). Average glucose was increased from group 1 to 4 (FIG. 3a). The data of group 1 and 2 were overlapped, while the data of group 3 and 4 were divided. Similarly, HbA1c value was increased from group 1 to 4 (FIG. 3b). .The mean value of 4 groups increased from group 1 to 4 , while the distribution of the data was rather overlapped in group 1-4.
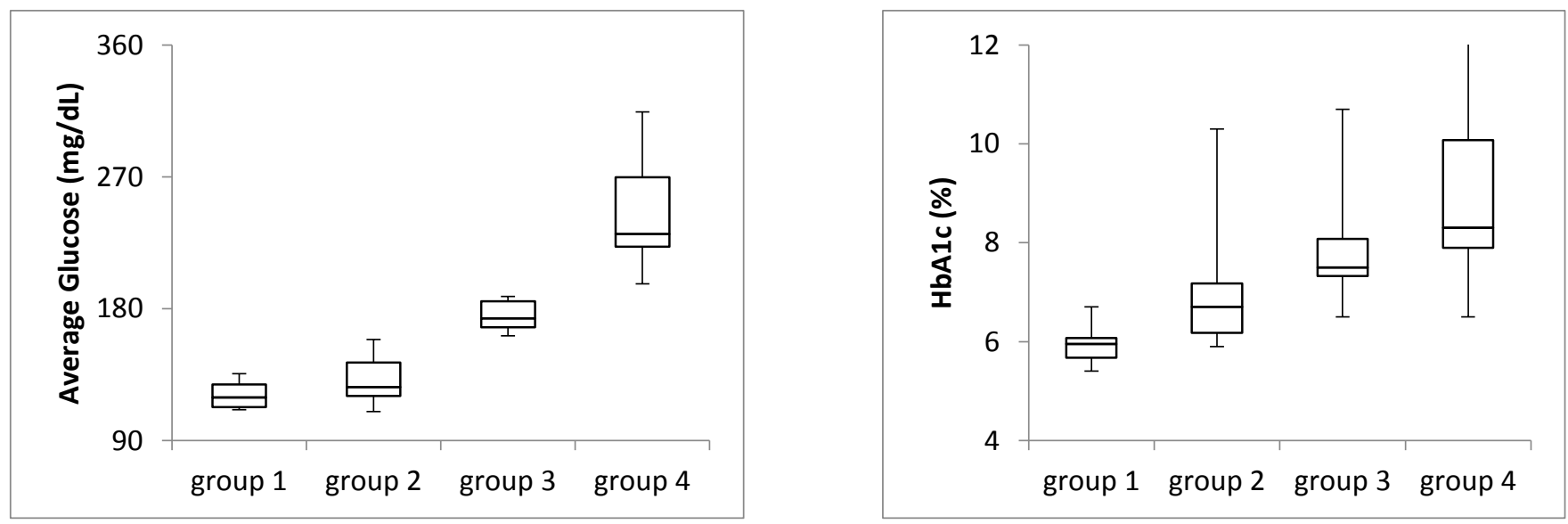

FIG. 3. Comparison of average glucose and HbA1c in 4 groups. FIG. 3a. Average Glucose was increased from group 1 to 4, FIG. 3b. HbA1c value was increased from group 1 to 4. 
www.yumedtext.com | August-2018

\subsection{Comparison of HOMA-R and HOMA- $\beta$ in 4 groups}

Regarding HOMA-R, the value in group 1,2,3 were rather overlapped, and that of group 4 showed higher level than other groups (FIG. 4a). Regarding HOMA- $\beta$, the value in group 1,2,3,4 showed rather divided distribution, and their levels was decreased from group 1 to 4 (FIG. 4b).
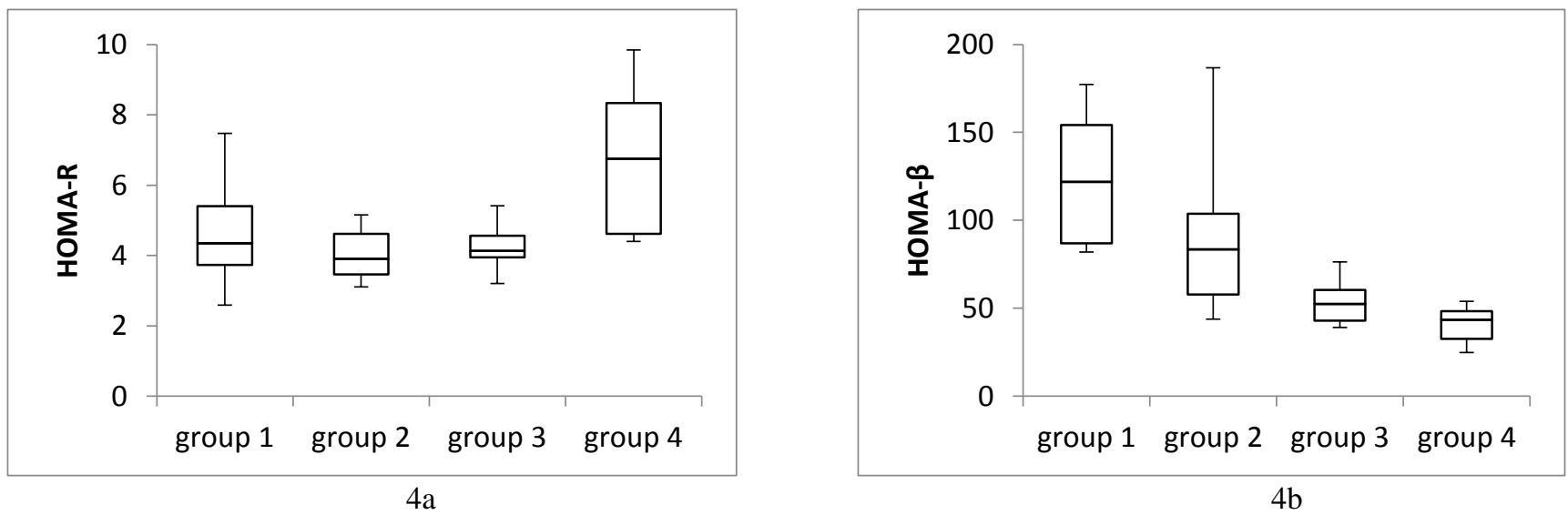

FIG. 4. Comparison of HOMA-R and HOMA- $\beta$ in 4 groups. FIG 4a. The value of HOMA-R in group 4 tended to be higher level than other groups, FIG. $4 \mathrm{~b}$. The value of HOMA- $\beta$ decreased from group 1 to 4 .

\subsection{Correlation of M value and HOMA}

There was significant correlation between $M$ value and HOMA-R ( $\mathrm{p}<0.05)$ (FIG. 5a). Similarly, there was significant correlation between M value and HOMA- $\beta$ ( $<<0.01$ ) (FIG. 5b). HOMA- $\beta$ showed higher correlation coefficient than HOMAR.

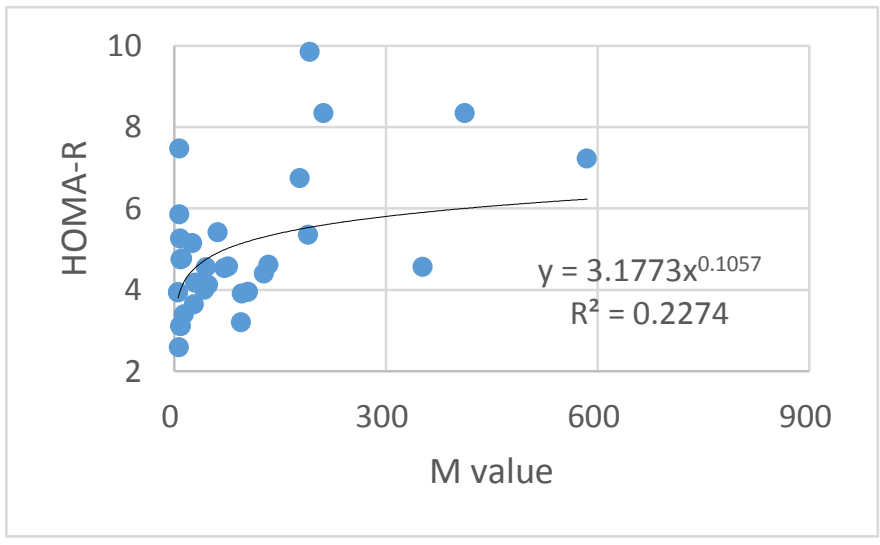

$5 \mathrm{a}$

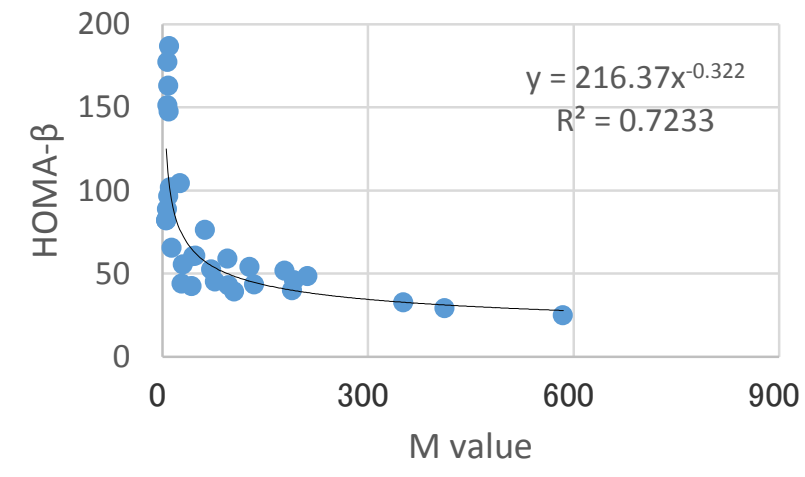

$5 b$

FIG. 5. Correlation of M value and HOMA. FIG. 5a. There was significant correlation between $M$ value and HOMA-R ( $p<0.05$ ), FIG. 5b. There was significant correlation between M value and HOMA- $\beta$ ( $p<0.01)$. HOMA- $\beta$ showed higher correlation coefficient than HOMA-R. 
www.yumedtext.com | August-2018

\section{Discussion}

As to various problems of diabetes, adequate management and treatment have been crucial for years. Especially, nutritional therapy would be the fundamental and important.

For long years, diabetes has been increasing worldwide and became medical, social and economic problems [21]. The cause of diabetic complications are from elevated blood glucose, that is, persisting hyperglycemia [22,23]. As to microvascular complications, four possible mechanisms influencing the biochemical changes were found. They are formation of advanced glycation end products (AGEs), increased flux of glucose and other sugars through the polyol pathway, activation of protein kinase $\mathrm{C}(\mathrm{PKC})$ isoforms and increased flux through the hexosamine pathyway [24,25].

Hyperglycemia involves blood glucose fluctuations including insulin secretion. In this study, we investigated type 2 diabetes from several aspects of glucose variability and examined the cases of T2DM that showed fasting IRI values greater than 10 $\mu \mathrm{U} / \mathrm{mL}$. The reason would be from our continuing clinical research about CR and LCD. Patients with T2DM show wide variety of heterogeneous situation in several biomarkers. Then, it would be not adequate to analyze all diabetic patients together, because there are various different insulin resistance and pancreas function.

As comparative study, we have unpublished data which are related to these investigations. We have lots of data from diabetic patients, in which subjects were divided by the value of IRI. The categorization by IRI was 5 or less vs 5-10 vs more than 10 in 3 groups. The comparative data in each group was 0-3 vs 0-7 vs 3-10 for HOMA-R, and 0-50 vs 19-90 vs 25-177 for HOMA-beta (unpublished data) [26]. Furthermore, some correlations among biomarkers have been investigated. Several correlation and distribution patterns among these showed various situations. Accumulation of these research data would become the fundamental data in this area.

As to the background of the cases in this study, the average of subjects is 57 years old in median. The half belongs to $49-65$ years old, indicating the research of T2DM from middle age to high age period. Further, the characteristic point would be the existence of mild insulin resistance according to the value of fasting IRI.

In response to LCD from day 2 to day 14, there were decreased values in average blood glucose, M value and triglyceride. Several reports were found about the efficacy of LCD for improving glucose variability and triglyceride [27]. Our results were consistent with previous reports.

As for the changes of lipid profile against LCD, some findings were reported. Triglyceride level usually decreases for a certain period of LCD. On the other hand, HDL has been said to show a slight upward trend after several months. LDL has not have a certain trend, including constant, decreasing or increasing depending on the case. In this study, the values in HDL and LDL did not show remarkable changes in 2 weeks.

Furthermore, the usefulness of $\mathrm{M}$ value was clarified in this study. $\mathrm{M}$ value represents two aspects of glucose variability, which are mean blood glucose a day and also MAGE. Compared with the changes in glucose level, the range of M value is 
www.yumedtext.com | August-2018

rather wide, and then it can be useful for clinical research in the case of correlation and/or comparison among several biomarkers.

When examined separately in 4 groups, the mean blood glucose level clearly decreased in all groups. On the other hand, by the scale of $\mathrm{M}$ value, the degree of decrease was somewhat lower in group 1. The reason for this is the definition and calculation method of $\mathrm{M}$ value, where the standard level of blood sugar level is based on $120 \mathrm{mg} / \mathrm{dL}$ [18,19]. For example, as the blood glucose decrease from $120 \mathrm{mg} / \mathrm{dL}$ to $100 \mathrm{mg} / \mathrm{dL}$, the calculated level of M value will increase. Actually in group 1, average blood glucose was $119 \mathrm{mg} / \mathrm{dL}$ on day 2 and $104 \mathrm{mg} / \mathrm{dL}$ on day 14 . According to the formula equation of M value, the level of $\mathrm{M}$ value has not decreased.

On the other hand in group 2-4, as the average glucose decrease from day 2 to 14, the level of M value obviously decreased. Furthermore, the degree of decrease ratio seemed to be larger in the order of group 2-4.

Comparing the average blood glucose level and the $M$ value in this way, the $M$ value does not change so much when the average blood glucose level is in the range of $110 \mathrm{mg} / \mathrm{dL}$ to $130 \mathrm{mg} / \mathrm{dL}$. However, above that, as the blood glucose level rises, the $\mathrm{M}$ value increases to a great degree [28,29].

Consequently, in various disease states, $\mathrm{M}$ value seems to show a larger change than that of mean blood glucose level. $\mathrm{M}$ value can include two important clinical significance. Those are mean blood glucose level and MAGE, with expression of one numerical value. For this characteristic point, $M$ value seems to have benefits in the usage of clinical research [30,31].

For the treatment of LCD, the mean blood glucose level decreased in 12 days. Since the regression curve is $y=0.48 \mathrm{x}+45.9$, we can speculate that if the glucose is $200 \mathrm{mg} / \mathrm{dL}$ on day 2 , glucose level on day 14 is calculated as $142 \mathrm{mg} / \mathrm{dL}$.

The regression curve of the $M$ value between day 2 and day 14 is $y=0.16 x+1.2$. From the fact that the coefficient of the linear equation is 0.16 , the latter numerical value is remarkably reduced from the previous value. Actually, LCD showed remarkable effects on glucose and $\mathrm{M}$ value during only 12 days [29,32,33]. This means that both mean glucose level and MAGE have improved. From mentioned above, $M$ value seems to be useful for clinical research study on blood glucose fluctuation.

We compared the mean blood glucose and HbA1c data in 4 groups. In the former, various data seemed to be divided into three part distribution, which are, group $1 \& 2$, group 3, group 4. The reason for this was probably because the data was calculated from 7 daily profile of glucose on the day studied, including average glucose and MAGE.

On the other hand, in the latter, the value of median HbA1c rose from group 1 to 4 , but the distribution of the whole was overlapped in group 1-4. The reason for this would be from that HbA1c represents glucose variability in the past 1-2 months. HbA1c does not represent glucose variability on the day tested, probably with low relationship each other. 
www.yumedtext.com | August-2018

Distribution was studied in 4 groups for HOMA-R and HOMA- $\beta$. As a result, in HOMA-R, there was rather wide overlapped distribution in groups 1, 2, 3, but only group 4 was situated rather apart from others.

On the other hand, in HOMA- $\beta$, distribution was found to be divided in 4 groups. Especially in groups 3 and 4, the HOMA- $\beta$ value was obviously low, suggesting a situation in which the decrease of insulin secretion ability is lower than that of group 1 and 2.

As to the correlation of $M$ value, HOMA-R showed a significant positive correlation $(\mathrm{p}<0.01$ ), and HOMA- $\beta$ showed a significant negative correlation $(\mathrm{p}<0.01)$. Among them, the correlation coefficient was higher in the latter HOMA- $\beta$. The reason for this would be that $\mathrm{M}$ value includes mean blood glucose and MAGE with close relationship with insulin secretion, and that HOMA- $\beta$ represents pancreas function, leading to higher correlation coefficient in the latter $[26,34,35]$.

Concerning the limit of the study, we can speculate some points associated with our previous continuing investigations. T2DM patients have showed rather wide range of fasting IRI in clinical studies $[11,36]$. Probably, it is not adequate to take all cases into the research as a whole. Then, we have tried to selected cases by IRI values associated with similar pathophysiological background in diabetic patients. We are to plan develop this investigation successively.

Currently obtained results would become the reference and basal data in this field of research. For future study, we will compare the detail characteristic points in these groups, and will explore other related biomarkers, such as the response of glucose and IRI to standard meal of CR or LCD, urinary C-peptide, ketone bodies in blood, related study of liver and renal function tests and so on [13,37].

\section{Conclusion}

In summary, we investigated 40 patients with T2DM. Diabetes related biomarkers including HbA1c, glucose, IRI, HOMA-R, HOMA- $\beta$ were measured and correlations were investigated. In response to LCD for 2 weeks, average glucose level and M value were remarkably decreased and studied in detail for categorization in to 4 groups. These results suggest that LCD would be clinically effective for T2DM and these findings would be reference and basal data in this research, leading to further research development.

\section{Acknowledgement}

Regarding current article, some part of the content was presented at annual congress of Japan Diabetes Society (JDS) Conference, Tokyo, 2018. The authors would like to express gratitude all staffs and patients for the cooperation.

\section{Conflicts Of Interest}

The authors state that they have no conflicts of interest concerning to this paper. 


\section{REFERENCES}

1. American Diabetes Association. Pharmacologic Approaches to Glycemic Treatment: Standards of Medical Care in Diabetes-2018. Diabetes Care. 2018;41(1):S73-S85.

2. American College of Physicians. Clinical Guidelines and Recommendations. 2017. http://www.acponline.org/clinical-information/guidelines

3. International Diabetes Federation (IDF). Standards of Medical Care in Diabetes-2015. Diabetes Care. 2015;38(1):S1-S94.

4. Bando H. Statement for Diabetes Guideline Has Been on Discussion for Future Better Lives. J Endocrinol Thyroid Res. 2018;3(4): 555616.

5. Bernstein RK. Dr. Bernstein's Diabetes Solution. New York: Little Brown and Co., USA; 1997.

6. Shai I, Schwarzfuchs D, Henkin Y, et al. Dietary Intervention Randomized Controlled Trial (DIRECT) Group. Weight Loss with a Low-Carbohydrate, Mediterranean, or Low-Fat Diet. N Engl J Med. 2008;359(3):229-41.

7. Schwarzfuchs D, Golan R, Shai I. Four-year follow-up after two-year dietary interventions. N Engl J Med. 2012;367(14):1373-4.

8. Accurso A, Bernstein RK, Dahlqvist A, et al. Dietary carbohydrate restriction in type 2 diabetes mellitus and metabolic syndrome: time for a critical appraisal. Nutr Metab (Lond). 2008;5:9.

9. Meng Y, Bai H, Wang S, et al. Efficacy of low carbohydrate diet for type 2 diabetes mellitus management: A systematic review and meta-analysis of randomized controlled trials. Diabetes Res Clin Pract. 2017;131:124-31.

10. Ebe K, Ebe Y, Yokota S, et al. Low Carbohydrate diet (LCD) treated for three cases as diabetic diet therapy. Kyoto Medi Association J. 2004;51:125-29.

11. Bando H, Ebe K, Muneta T, et al. Effect of low carbohydrate diet on type 2 diabetic patients and usefulness of Mvalue. Diabetes Res Open J. 2017;3(1):9-16.

12. Muneta T, Kawaguchi E, Nagai Y, et al. Ketone body elevation in placenta, umbilical cord, newborn and mother in normal delivery. Glycative Stress Res. 2016;3(3):133-40.

13. Ebe K, Bando H, Yamamoto K, et al. Daily carbohydrate intake correlates with HbA1c in low carbohydrate diet (LCD). J Diabetol. 2018;1(1):4-9.

14. Bando H, Ebe K, Muneta T, et al. Clinical Effect of Low Carbohydrate Diet (LCD): Case Report. Diabetes Case Rep. 2017;2(2):124.

15. Japan Diabetes Association. Diabetes clinical practice guidelines Based on scientific evidence. 2013.

16. Schlichtkrull J, Munck O, Jersild M. The M-value, an index of blood sugar control in diabetics. Acta Med Scand. 1965;177:95-102.

17. Service FJ, Molnar GD, Rosevear JW, et al. Mean amplitude of glycemic excursions, a measure of diabetic instability. Diabetes. 1970;19(9):644-55.

18. Molnar GD, Taylor WF, Ho MM. Day-to-day variation of continuously monitored glycaemia: A further measure of diabetic instability. Diabetologia. 1972;8(5):342-8.

19. Moberg E, Kollind M, Lins PE, et al. Estimation of blood-glucose variability in patients with insulin-dependent diabetes mellitus. Scand J Clin Lab Invest. 1993;53(5):507-14.

20. Yanai H. Four step excel statistics. 4th ed. Tokyo: Seiun-sha Publishing Co.Ltd, Japan; 2015.

21. World Health Organization. Global report on diabetes. WHO, Geneva, Switzerland. 2016. 
www.yumedtext.com | August-2018

22. Ojo O. An overview of diabetes and its complications. Diabetes Res Open J. 2016;2(2):e4-e6.

23. Defronzo RA. Banting Lecture. From the triumvirate to the ominous octet: a new paradigm for the treatment of type 2 diabetes mellitus. Diabetes. 2009;58(4):773-95.

24. Williams G, Pickup JC. Handbook of Diabetes. 3rd ed. New Jersey: Blackwell Publishing, USA; 2004.

25. Dunning T. Care of People with Diabetes: A Manual of Nursing Practice. New Jersey: Wiley Blackwell, USA; 2014.

26. Matthews DR, Hosker JP, Rudenski AS, et al. Homeostasis model assessment: insulin resistance and beta-cell function from fasting plasma glucose and insulin concentrations in man. Diabetologia. 1985;28(7):412-9.

27. Feinman RD, Pogozelski WK, Astrup A, et al. Dietary carbohydrate restriction as the first approach in diabetes management: Critical review and evidence base. Nutrition. 2015;31(1):1-13.

28. DeVries JH. Glucose Variability: Where It Is Important and How to Measure It. Diabetes. 2013;62(5):1405-8.

29. Fritzsche G, Kohnert KD, Heinke P, et al. The Use of a Computer Program to Calculate the Mean Amplitude of Glycemic Excursions. Diabetes Technol Therap. 2011;13(3):319-25.

30. Borg R, Kuenen JC, Carstensen B, et al. $\mathrm{HbA}_{1}(\mathrm{c})$ and mean blood glucose show stronger associations with cardiovascular disease risk factors than do postprandial glycaemia or glucose variability in persons with diabetes: the A1C-Derived Average Glucose (ADAG) study. Diabetologia. 54(1):69-72.

31. Kilpatrick ES, Rigby AS, Atkin SL. Effect of Glucose Variability on the Long-Term Risk of Microvascular Complications in Type 1 Diabetes. Diabetes Care. 2009;32(10):1901-3.

32. Hill NR, Oliver NX, Choudhary P, et al. Normal Reference Range for Mean Tissue Glucose and Glycemic Variability Derived from Continuous Glucose Monitoring for Subjects Without Diabetes in Different Ethnic Groups. Diabetes Technol Ther. 2011;13(9):921-8.

33. Bando H, Ebe K, Muneta T, et al. Effect of low carbohydrate diet on type 2 diabetic patients and usefulness of Mvalue. Diabetes Res Open J. 2017;3(1):9-16.

34. Cersosimo E, Solis-Herrera C, Trautmann ME, et al. Assessment of pancreatic $\beta$-cell function: review of methods and clinical applications. Curr Diabetes Rev. 2014;10(1):2-42.

35. Veijola R, Koskinen M, Helminen O, et al. Dysregulation of glucose metabolism in preclinical type 1 diabetes. Pediatr Diabetes. 2016;17(22):25-30.

36. Bando H, Ebe K, Muneta T, et al. Study on responses of C-peptide and blood glucose for Carbohydrate $70 \mathrm{~g}$ in diabetes. Endocrinol Metab. 2018;2(2):110.

37. Qu C, Zhou X, Yang G, et al. The natural logarithm of zinc- $\alpha 2$-glycoprotein/HOMA-IR is a better predictor of insulin sensitivity than the product of triglycerides and glucose and the other lipid ratios. Cytokine. 2016;79:96-102. 